\title{
Caracterização da postura corporal e do padrão respiratório em crianças
}

asmáticas

\author{
Eduardo Matias dos Santos Steidl*, Fernanda Freire Friedrich*, \\ Vívian da Pieve Antunes ${ }^{\star \star *}$, Cláudia Regina Beuter***
}

\begin{abstract}
Resumo: Objetivo - Caracterizar as principais alterações posturais e o tipo de padrão respiratório encontrado em uma amostra de crianças asmáticas. Metodologia - 0 estudo caracterizou-se como uma série de casos prospectivos. Após aprovação pelo Comitê de Ética em Pesquisa da instituição e aceite do termo de consentimento pelos pais e responsáveis, 13 crianças que foram submetidas à avaliação postural subjetiva e do tipo de padrão respiratório, onde os dados foram apresentados por meio de estatística descritiva. Resultados - A média de idade foi de 8,75 $\pm 1,81$ anos, o padrão apical foi mais prevalente $(61,35 \%)$ as principais alterações posturais encontradas foram na cervical (inclinação - 38,46\%, protração - 46,15\%), ombros (protração - 76,92\%, anteversão - 15,38\%), quadril em anteversão - 100\%, joelhos (rotação interna - 38,46\%, hiperextensão - 46,15\%), hiperlordose de coluna lombar $(84,61 \%)$ e retificação torácica $(46,15 \%)$. Conclusão - Além do comprometimento pulmonar, a asma pode desencadear alterações posturais e do padrão respiratório em crianças.
\end{abstract}

Descritores: Postura; Mecânica Respiratória; Asma.

\section{Characterization of body posture and breathing pattern in asthmatic} children

\begin{abstract}
Objective - To characterize the main postural changes and the type of breathing pattern found in a sample of asthmatic children. Method - The study was characterized as a prospective case series. After approval by the Ethics in Research Committee and accept the consent of parents and guardians, 13 children who underwent subjective postural assessment and the type of breathing pattern, where the data were presented using descriptive statistics. Results - The mean age was 8.75 \pm 1.81 years, the standard apical prevailed in $61.35 \%$ of children and major postural changes were found in the cervical (slope - $38.46 \%$, protraction - $46.15 \%$ ), shoulders (protraction - $76.92 \%$, anteversion - 15.38\%), hip anteversion - 100\%, knees (internal rotation - 38.46\%, hyperextension $46.15 \%)$, lumbar hyperlordosis (84.61\%) and thoracic rectification (46.15\%). Conclusion - In addition to the pulmonary, asthma can trigger postural and breathing pattern in children.
\end{abstract}

Descriptors: Posture; Respiratory Mechanics; Asthma.

\footnotetext{
* Graduado em Fisioterapia pelo Centro Universitário Franciscano (UNIFRA), Santa Maria, RS, Brasil. Mestrando em Distúrbios da Comunicação Humana na Universidade Federal de Santa Maria (UFSM), Santa Maria, RS, Brasil.

** Graduado em Fisioterapia pelo Centro Universitário Franciscano (UNIFRA), Santa Maria, RS, Brasil.

*** Mestranda em Distúrbios da Comunicação Humana na Universidade Federal de Santa Maria (UFSM), Santa Maria, RS, Brasil.

**** Mestre em Distúrbios da Comunicação Humana pela Universidade Federal de Santa Maria (UFSM), Santa Maria, RS, Brasil.
} 


\section{Introdução}

A asma pode ser definida como uma doença pulmonar inflamatória crônica, caracterizada pela hiperreatividade das vias aéreas inferiores e limitação variável ao fluxo aéreo, sendo reversível ou não, com o uso de medicamentos, como os broncodilatadores ${ }^{1,2}$, causando episódios de dispneia, pressão torácica e tosse, geralmente a noite ou pela manhã ${ }^{3}$. Ela resulta de uma interação entre fatores genéticos, exposição ambiental e alguns fatores específicos que levam ao desenvolvimento e à manutenção dos sintomas ${ }^{4}$.

A asma brônquica é uma das afecções respiratórias ainda carentes de solução, que vem sendo descrita nas últimas décadas, assumindo valores epidêmicos ${ }^{1}$. Pessoas de diversos países em todas as faixas etárias são acometidas por essa doença que pode cursar com crises muito graves e algumas vezes se tornar fatal. Estudos epidemiológicos no Brasil são poucos, e a verdadeira dimensão da doença é desconhecida, onde um dos poucos estudos demonstra que $25 \%$ da população seja asmática e as crianças as mais atingidas ${ }^{5}$.

A doença não controlada pode tornar-se crônica com limitação permanente do fluxo aéreo, levando à limitação física e social significativa, comprometendo a qualidade de vida, além de relacionar-se com alterações posturais significativas ${ }^{6,7}$. Isto se explica pelo recrutamento excessivo dos músculos inspiratórios acessórios e expiratórios, em resposta a obstrução ao fluxo aéreo, o que leva a uma hipertrofia adaptativa ${ }^{8,9}$, resultando em encurtamento e perda da sua flexibilidade ${ }^{10-12}$.

Uma mecânica respiratória insatisfatória resulta em um bloqueio inspiratório, com a diminuição do volume expiratório e da capacidade inspiratória. Porém, a biomecânica da caixa torácica não funciona de forma isolada, estando inserida numa mecânica corporal, onde qualquer desequilíbrio respiratório trará reflexos negativos sobre a organização da mesma ${ }^{8,11,12}$.

Nesta visão, é cada vez maior a convicção de que o melhor caminho é a conjugação de diferentes esforços e a participação de diversos profissionais. Nessa linha, ocupa espaço cada vez maior a atuação do profissional fisioterapeuta ${ }^{13}$ que na prática clínica, vem observando a presença de alterações na postura comuns às crianças asmáticas, como a hiperlordose lombar que isoladamente poderia comprometer a mecânica ventilatória, gerando um deslocamento das vísceras, a retificação e a diminuição da efetividade do diafragma ${ }^{14}$.

Baseando-se nessas premissas o presente estudo teve por objetivo caracterizar a postura corporal e o padrão respiratório de crianças asmáticas, a fim de evidenciar as principais estruturas posturais acometidas e o padrão respiratório predominante.

\section{Metodologia}

A pesquisa caracterizou-se como série de casos prospectivos. $O$ estudo atendeu ás normas da resolução 196/96-CNS e foi aprovado pelo Comitê de Ética em Pesquisa (CEP) do Centro Universitário Franciscano, sob registro 234.2011.12. Após aprovação do projeto de

Saúde (Santa Maria), v.39, n.1, p. 131-138, respiratório em crianças asmáticas 132 pesquisa pelo CEP e anuência do Termo de Consentimento Livre e Esclarecido (TCLE) pelos pais ou responsáveis o projeto foi executado.

A população foi composta por dezesseis crianças, provenientes da cidade de Santa Maria - RS, que apresentassem diagnóstico clínico de asma brônquica. Para a inclusão das 
crianças, foram utilizados os seguintes critérios: diagnóstico clínico de asma atópica, faixa etária de sete a doze anos, não serem obesas (Índice de Massa Corporal - IMC $\geq 24,4$ $\left.\mathrm{kg} / \mathrm{m}^{2}\right)^{15}$, sem alterações neurológicas e ortopédicas diagnosticadas, bem como aceite do TCLE pelos pais ou responsáveis.

As crianças foram avaliadas individualmente, com a presença dos pais ou responsáveis, em um único encontro. Foi aplicada uma ficha de coleta de dados elaborada pelos pesquisadores, contendo dados pessoais e da história clínica, bem como os sintomas e frequência de exacerbação das crises, número de vezes em que as crianças haviam sido hospitalizadas, uso de medicações e presença de doenças respiratórias associadas. Em seguida foi realizada a aferição do peso e altura para o cálculo do IMC, conforme proposto por Damasceno et al. ${ }^{15}$.

Logo após foi aplicada uma ficha de avaliação postural ${ }^{16}$, sempre pelo mesmo observador, onde a criança permaneceu em bipedestação, fazendo uso de trajes de banho (meninos, sunga ou calção; meninas, biquíni), em sala climatizada, sendo avaliados os itens relacionados à postura da cabeça, ombros, quadril, coluna vertebral, joelhos e pés, em vista anterior, posterior e perfil. As avaliações ocorreram tanto no período da manhã quanto da tarde, de acordo com o horário disponível dos pais e das crianças.

O padrão respiratório foi avaliado através do Índice Diafragmático (ID) com uso de fita métrica, o qual é determinado pelas alterações nas dimensões ântero-posterior do tórax e abdome durante a fase inspiratória e expiratória, onde a respiração é realizada profundamente ${ }^{17}$. As medidas da movimentação do abdome foram mensuradas ao nível da cicatriz umbilical e do tórax ao nível do $4^{\circ}$ espaço intercostal.

Para análise estatística descritiva, os dados foram tabulados em uma planilha do programa Microsoft Excel 2007, onde foram utilizadas as médias, desvio padrão e porcentagem simples.

\section{Resultado e discussão}

Após a aplicação da ficha de coleta de dados, foram excluídas três crianças por serem obesas (IMC 25,3 kg/m²; $26,2 \mathrm{~kg} / \mathrm{m}^{2} ; 25,7 \mathrm{~kg} / \mathrm{m}^{2}$ ), compondo uma amostra de treze crianças. Os dados encontrados em relação ao perfil das crianças avaliadas podem ser visualizados na tabela abaixo (Tabela 1): 
Tabela 1: Caracterização do perfil das crianças avaliadas

\begin{tabular}{|c|c|c|c|c|c|}
\hline Criança & Idade & Sexo & Sintomas & $\begin{array}{c}\text { Dias de } \\
\text { exacerbação }\end{array}$ & $\begin{array}{l}\text { Padrão } \\
\text { respiratório }\end{array}$ \\
\hline 1 & 8 & M & Dispneia, sibilância e presença de secreção. & 3 & Misto \\
\hline 2 & 7 & $\mathrm{~F}$ & Dispneia, sibilância e presença de secreção. & 4 & Misto \\
\hline 3 & 9 & $\mathrm{~F}$ & Dispneia, sibilância e presença de secreção. & 7 & Apical \\
\hline 4 & 10 & M & Dispneia, sibilância e presença de secreção. & 3 & Apical \\
\hline 5 & 7 & $\mathrm{~F}$ & Dispneia, sibilância e presença de secreção. & 7 & Misto \\
\hline 6 & 12 & $\mathrm{~F}$ & Tosse, dispneia & 2 & Apical \\
\hline 7 & 12 & $\mathrm{~F}$ & Tosse, dispneia, sibilância e secreção & 2 & Misto \\
\hline 8 & 7 & $\mathrm{~F}$ & Sibilos, secreção & 2 & Apical \\
\hline 9 & 8 & $\mathrm{~F}$ & Tosse, dispneia & 3 & Apical \\
\hline 10 & 7 & M & Tosse, dispneia, sibilância e secreção & 4 & Apical \\
\hline 11 & 9 & M & Tosse, dispneia, sibilância e secreção & 4 & Misto \\
\hline 12 & 9 & M & Tosse, dispneia, sibilância e secreção & 5 & Apical \\
\hline 13 & 6 & $\mathrm{~F}$ & Tosse, dispneia, sibilância e secreção & 4 & Misto \\
\hline
\end{tabular}

$M=$ masculino; $F=$ feminino.

As crianças avaliadas foram escolares, na faixa etária de sete a doze anos, com média de idade de $8,75 \pm 1,81$ anos, onde cinco crianças foram do sexo masculino $(38,46 \%)$ e oito crianças do sexo feminino $(61,54 \%)$, sendo todas classificadas com asma do tipo atópica.

De acordo com o questionário de coleta de dados, todas as crianças apresentaram os sintomas característicos da asma (tosse, dispneia, sibilos e a presença de secreção) ao entardecer e a noite, fazendo uso de broncodilatador diariamente (salbutamol) uma vez ao dia pela manhã, prevalecendo em $53,84 \%(n=7)$ das crianças a presença dos sintomas em torno de três a quatro dias da semana, com presença dos sintomas mensalmente.

Em relação ao tipo de padrão respiratório, oito crianças $(61,35 \%)$ apresentaram padrão respiratório apical (ID $0,38 \pm 0,05)$ e cinco $(38,46 \%)$ foram classificadas como padrão do tipo misto (ID 0,5).

Os dados relacionados com as alterações posturais encontram-se agrupados na tabela abaixo (Tabela 2): 
Tabela 2: Postura corporal das crianças avaliadas

\begin{tabular}{ccc}
\hline Itens avaliados & Caracterização & (n) / \% \\
\hline Cervical & Inclinação & $5 / 38,46 \%$ \\
& Protração & $6 / 46,15 \%$ \\
Ombros & Protração & $10 / 76,92 \%$ \\
& Anteversão & $2 / 15,38 \%$ \\
Quadril & Anteversão & $13 / 100 \%$ \\
Joelhos & Rotação Interna & $5 / 38,46 \%$ \\
Coluna lombar & Hiperextensão & $6 / 46,15 \%$ \\
Coluna Torácica & Retificação & $6 / 46,15 \%$ \\
\hline
\end{tabular}

De acordo com a tabela acima, pode-se observar que as estruturas de maiores alterações foram os ombros, quadril, coluna vertebral e joelhos.

$\mathrm{Na}$ amostra estudada, o gênero mais acometido pela asma foi o feminino (53,84\%), porém, a diferença da prevalência da asma entre gêneros é controversa na literatura e estudos evidenciam uma prevalência maior no gênero masculino durante a fase escolar, e na adolescência o feminino ${ }^{18,19}$. A asma atópica é o tipo mais prevalente na população asmática, onde cerca de $80 \%$ a $90 \%$ são sensíveis a pelo menos um alérgeno ambiental20, fato também verificado no presente estudo.

Em relação à sintomatologia, as crianças avaliadas apresentaram sibilância, dispneia, aperto no peito e tosse, principalmente à noite e ao despertar, como é apresentado na literatura ${ }^{21,22}$. A exacerbação da doença ao entardecer relaciona-se ao ciclo circadiano, que influencia diretamente a produção de cortisol, onde nesse período do dia ocorre uma queda em sua produção. Conforme o II Consenso Brasileiro no Manejo da asma ${ }^{23}$, durante a noite ocorre redução dos níveis de catecolaminas e cortisol plasmático, permitindo uma maior expressão linfocitária e a ativação de uma cascata inflamatória nas vias aéreas, facilitando a exacerbação dos sintomas.

Quanto ao padrão respiratório dos pacientes, o presente estudo demonstrou alterações, evidenciando que sete crianças $(53,84 \%)$ utilizavam o padrão apical.

Pode-se verificar que o padrão respiratório apical apresentou-se na maioria dos pacientes asmáticos, o que está de acordo com a literatura. No estudo de Ratto ${ }^{24}$, o autor compara o padrão respiratório com a postura de crianças asmáticas, demonstrando que algumas alterações torácicas e posturais são causadas pela asma, pois a mecânica de funcionamento do tórax é importante e as alterações respiratórias, segundo sua origem, podem modificar essa mecânica e/ou funcionamento fisiológico do pulmão.

Em um estudo de Marcelino et al. ${ }^{25}$, os autores relatam que os asmáticos apresentam um padrão respiratório apical, podendo ser causado pela desvantagem da musculatura respiratória, através da dificuldade na expiração, ocorrendo o aprisionamento de ar e conseqüentemente a hiperinsuflação pulmonar, que não permite um amplo movimento do diafragma, fazendo com que este músculo fique numa posição mais plana e apresente fraqueza muscular ${ }^{26}$.

Rev. Saúde (Santa Maria), v.39, n.1, p. 131 . 138, Jan./Jul. 2013 Steidl, E. et al. 
Kapandji27 afirma que as doenças pulmonares obstrutivas como a asma provocam alterações na mecânica respiratória, interferindo diretamente na postura corporal. A repetição das crises, com o aumento do volume residual e o uso exacerbado da musculatura acessória, gera restrição na mobilidade torácica e alterações no tórax e na coluna vertebral como um todo. No entanto, a combinação de alterações tóraco-vertebrais é comum e, muitas vezes, é difícil apontar com precisão se as disfunções pulmonares provocaram as alterações posturais ou se foram essas que agravaram a disfunção pulmonar. Além disso, na criança, o tórax, por sua forma, função e elasticidade, é facilmente deformável, o que explica porque as alterações posturais são menos freqüentes nos adultos que nas crianças.

$\mathrm{Na}$ avaliação postural dos escolares participantes do estudo constatou-se que as principais alterações relacionam-se com coluna vertebral, joelhos, ombros e quadril.

Os resultados encontrados em relação à posição da cervical são controversos na literatura. Em um estudo realizado por Mellin ${ }^{28}$, ao analisar as curvaturas da coluna vertebral de 35 adolescentes asmáticos e não asmáticos, observou que o grupo de asmáticos não possuía aumento das curvaturas em comparação aos não asmáticos. Da mesma maneira, Azevedo et al..$^{14}$, ao analisarem a postura de 10 crianças asmáticas comparadas com um grupo de 26 não-asmáticas, por meio dos instrumentos palm meter e da régua flexível, demonstraram que as crianças diagnosticadas com asma não diferiram significativamente do grupo de crianças não-asmáticas em relação ao grau de curvatura da lordose lombar.

Em contrapartida, Belli et al. ${ }^{29}$ realizaram a avaliação da postura corporal estática de crianças asmáticas por meio da biofotogrametria computadorizada, onde participaram 30 crianças, 15 asmáticas e 15 não-asmáticas, de ambos os sexos, que foram submetidas a fotos em todos os ângulos. Posteriormente as fotos foram analisadas no software ALCimagem, encontrando desvios posturais nas crianças asmáticas, incluindo aumento da curvatura da cervical.

Os autores supracitados, no mesmo estudo, ao avaliarem as alterações em relação ao plano sagital, tais como, aumento da curvatura lombar e a hiperextensão dos joelhos obtiveram os mesmos resultados deste estudo.

Marques $^{30}$ afirma que a hiperlordose lombar isolada poderia provocar alguma modificação respiratória, com a exagerada projeção do abdome à frente, gerando o deslocamento das vísceras, a retificação do diafragma e a diminuição da efetividade do mesmo. Segundo o autor, na criança com asma, tais mudanças posturais são extremamente danosas por causarem grandes prejuízos à mecânica respiratória.

Os resultados encontrados em relação à postura dos ombros corroboram com estudos realizados por Robles-Ribeiro et al. ${ }^{31}$, onde os autores pesquisaram a postura corporal comparando com o pico de fluxo expiratório (PFE) de 19 crianças asmáticas, e com o estudo de Lopes et al. ${ }^{32}$ que avaliou 60 meninos com asma grave, onde ambos encontraram relações da doença com a postura dos ombros em protrusão.

Já em relação à postura do quadril, os dados encontrados vão de contrapartida aos resultados obtidos no estudo de Ricieri et al..$^{33}$, em que os autores realizaram a avaliação biofotogramétrica de 19 crianças asmáticas comparadas com 31 não asmáticas, e

Saúde (Santa Maria), v.39, n.1, p. 131-138,

Caracterização da postura corporal e do padrão respiratório em crianças asmáticas evidenciaram que não há alterações estatisticamente significativas referentes à posição do quadril. 


\section{Considerações Finais}

As principais assimetrias posturais encontradas neste estudo foram na coluna, ombros, joelhos e quadril e padrão respiratório do tipo apical. Dessa forma foi possível verificar que a asma acarreta alterações posturais significativas, evidenciando uma relação positiva entre as alterações pulmonares com a biomecânica corporal das crianças asmáticas avaliadas neste estudo.

Para tanto, sugere-se que sejam realizados novos estudos, a fim de comprovar a existência ou não da relação entre a asma, padrão respiratório e alterações posturais, visando à prevenção de possíveis distúrbios musculoesqueléticos desencadeados por uma função pulmonar anormal.

\section{Referências}

1. Paulin $E$, Favoreto $P$, Vidotto $C$. Benefícios da Fisioterapia respiratória na asma relato de um caso. Arq Ciências da Saúde 2001; 5(2): 149-154.

2. Taketomi EA, Marra SMG, Segundo GRS. Fisioterapia em asma: efeito na função pulmonar e em parâmetros imunológicos. Fit \& Person J 2005; 4(2): 97-100.

3. Diretrizes da Sociedade Brasileira de Pneumologia e Tisiologia Para o Manejo da Asma. J Bras Pneumol 2012; 38(supl 1): S1-S46.

4. Ferreira SP, Kirk K, Siqueira HR. Asma Brônquica. In: Silveira IC. O pulmão na prática médica. Rio de Janeiro: Biomédica; 2000.

5. Souza F. Morbidade e mortalidade por asma no Rio Grande do Sul no período de 1990 a 2001: existem diferenças entre os sexos?. [monografia]. Canoas: ULBRA; 2002.

6. Lopez M, Medeiros J. Semiologia Médica. Belo Horizonte: Atheneu; 1990.

7. Oliveira J, Freitas C. Fisioterapia pneumofuncional: perguntas \& respostas. Fortaleza: Fundação Edson de Queiroz; 2004.

8. Luce JM, Culver B. Respiratory muscles function in health and disease. Chest 1982; 81(1):82 -90.

9. Lavietes MH, Grocela JA, Maniatis T, Potulski F, Ritter AB, Sunderam G. Inspiratory muscle strength in asthma. Chest 1988; 93(5): $1043-1048$.

10. Cala SJ, Edyvean J, Engel LA. Chest wall and trunk muscle activity during inspiratory loading. J Applied Phys 1992; 6: $2373-2381$.

11. Souchard P. Le role de la reeducation posturale globale (RPG) dans les pathologies respiratoires. Rev Francaise Maladies Resp 2005; 22: $524-525$.

12. Baltar JA, Santos MSB, Silva HJ. A asma promove alterações na postura estática?. Rev Port Pneumol 2010; 16(3): 471-476.

13. Dweik R, Stoller JK. Doença pulmonar obstrutiva. In: Scalan, L.C.; Wilkins, L.R.; Stoller, K.J. Fundamentos da Fisioterapia Respiratória de Egan. $7^{\circ}$ ed. São Paulo: Manole; 2000. p. 439-478.

14. Azevedo VMGO, Rocha PM, Casaes GPE. et al. Alterações posturais em crianças asmáticas. Rev Paul Ped 2005; 23(3): 130-135.

Rev. Saúde (Santa Maria), v.39, n.1, p. 131 . 138, Jan./Jul. 2013.

Steidl, E. et al.

ISSN 2236-5843

137 
16. Santos A. Diagnóstico clínico postura: um guia prático. São Paulo: Summus; 2001.

17. Oliveira EA, Fernandes FE, Torquato JA. Verificação do padrão respiratório e força muscular respiratória em pacientes pediátricos com neoplasias encefálicas. Pediatr (São Paulo) 2009; 31(3): 143-151.

18. Amorim AJ, Daneluzzi JC. Prevalência de asma em escolares. J Pediatr 2001; 77(3): 197-202.

19. Cassol VE, Rizzato TM, Teche SP, Basso DF, Hirakata VN, Maldonado M. et al. Prevalence and severity of asthma among adolescents and their relationship and the body mass index. J Pneumol 2005; 27(5): 237242.

20. Solé D, Nunes ICC, Rizzo MCV, Naspitz CK. A asma na criança: classificação e tratamento. J Pediatr 1998; 74(supl.1): S48-S58.

21. Renz $H$. et al. $T(H) 1 / T(H) 2$ imune response profiles differ between atopic children in earsten and western Germany. J. Allergy Clin Immunol 2002; 42: 109-338.

22. Travensolo C, Rodrigues C. Qualidade de vida de um grupo de portadores de asma brônquica após um programa de Fisioterapia respiratória ambulatorial: relato de cinco casos. Revista Espaço para a Saúde 2006; 8: 28-33.

23. II Consenso Brasileiro no manejo da Asma. Asma Noturna. J Pneumol 1998; 24(4): 204-206.

24. Ratto OR. et al. Insuficiência respiratória. Rio de Janeiro: Atheneu; 1981.

25. Marcelino AMFC, Silva HJ. Papel da pressão inspiratória máxima na avaliação da força muscular respiratória em asmáticos: Revisão sistemática. Rev Port Pneumol 2010; 16(3): 463-470.

26. Lima EVNC, Lima WL, Nobre A, Santos AM, Brito LMO, Costa MRSR. Treinamento muscular inspiratório e exercícios respiratórios em crianças asmáticas. J Bras Pneumol 2008; 34(2): 552-558.

27. Kapandji IA. Fisiologia articular: esquemas comentados de mecânica humana: tronco e coluna vertebral. $5^{a}$.ed. São Paulo: Panamericana; 2000.

28. Mellin G. Increased spinal mobility in adolescents with bronchial asthma. J Rehab Med 1990; 22: 21-24.

29. Belli JFC, Oliveira AS, Bevilaqua-Grossi D. et al. Avaliação da postura corporal estática de crianças asmáticas através da fotogrametria computadorizada: um estudo preliminar. In: $14^{\circ}$ Simpósio Internacional de Iniciação Científica da USP; 2006.

30. Marques AP. Cadeias musculares: um programa para ensinar avaliação fisioterapêutica global. $1^{\text {a }}$ ed. São Paulo: Manole; 2000. p. 15-21.

31. Robles-Ribeiro P.; Ribeiro M, Lianza S. Relationship between peak expiratory flow rate and shoulders posture in healthy individuals and moderate to severe asthmatic patients. J Asthma 2005; 42: 783-786.

32. Lopes E, Fanelli-Galvani A, Prisco C, Gonçalves R, Jacob C, Cabral A, Martins M, Carvalho C. Assessment of muscle shortening and static posture in children with persistent asthma. Euro J Ped 2007; 166 : 715-721.

33. Ricieri DV, Costa JR, Filho NAR. Impacto da asma sobre a postura corporal de crianças entre 8 e 14 anos analisada pela biofotogrametria. Acta Fisiátrica 2008; 15(4): 214-219.

\section{Eduardo Matias dos Santos}

Endereço para correspondência - Rua André Marques, 617. Bairro Centro, Santa Maria, CEP: 97010 041, RS, Brasil.

Saúde (Santa Maria), v.39, n.1, p. 131-138,

Caracterização da postura corporal e do padrão respiratório em crianças asmáticas 138

ISSN 2236-

Recebido em 01 de outubro de 2012

Aprovado em 23 de julho de 2013. 